\title{
A fresh perspective on asthma
}

\author{
Recent advances in asthma research have altered views of how the disease is triggered and sustained. What remains \\ to be seen is whether this knowledge can be integrated into the clinic to alter the natural course of disease.
}

$\mathrm{T}$ - he global prevalence of asthma has markedly increased over the past 50 years, with an estimated 300 million people affected worldwide. Following the description of IgE and the T helper type $1\left(\mathrm{~T}_{\mathrm{H}} 1\right) / \mathrm{T}_{\mathrm{H}} 2$ hypothesis, scientists made considerable headway identifying pathways necessary to initiate and sustain allergic responses. However, clinical trials targeting these pathways met with mixed results, and, despite symptomatic control of the disease, individuals still develop chronic disease and remain refractory to therapies.

To identify recent advances that have affected understanding of asthma, Nature Medicine reached out to 50 basic and clinical researchers in asthma and asked them to highlight key advances and obstacles in the field. These recent developments are discussed in depth in seven reviews in this issue of Nature Medicine. We are indebted to the authors of these reviews and to the investigators surveyed for their insight into the field. What emerges is a picture of a highly heterogeneous disease, influenced by complex genetic and environmental effects, and an evolving understanding of the key cell types and soluble mediators that orchestrate the immune response.

One area of interest involves the intersection of microbial exposure with allergic disease, particularly as an early conditioning event in children. Although viral infections are known to exacerbate asthma, in a recent epidemiological study (N. Engl. J. Med. 364, 701-709, 2011), children who grew up on farms were exposed to a larger diversity of microbes and were protected from asthma. Though the specific microbes conferring protection were not identified, the culprits may lie within the gut. Commensal bacteria in the intestine can influence the development of several immune cells known to influence allergy, including basophils, invariant natural killer cells and T cells. Clinically, these findings dovetail with epidemiological studies linking disruptions in the gut microbiome with increased risk of allergic airway disease. Moreover, recent metagenomic analyses have revealed that the density and composition of commensal bacteria in the airways differ between subjects with asthma and control subjects (PLoS One 5, e8578, 2010). Though the role of these bacteria in asthma is unclear, these findings confirm the importance of early life exposure to microbes on the development of the immune system and allergic disease, and may point to a window of opportunity for intervention.

Epithelial cells lining the airways are the first line of defense against the entry of microbes and allergens. It is now clear that epithelial cells are not just passive participants responsible for walling off the body from the environment; instead, they respond to external cues and are directly activated by allergens through receptors of the innate immune system, releasing soluble mediators that orchestrate the subsequent adaptive immune response. These findings have overturned the idea that immune cells are the primary triggers of asthma and suggest that the airway epithelium has a sentinel role in bridging innate and adaptive immunity. Genetics studies have confirmed the importance of epithelial cells, as mutations in several epithelial cell-specific genes are associated with allergy and asthma. At present, it's unclear whether targeting innate receptors on epithelial cells can prevent the development of asthma in adulthood and what effect this might have on development of the immune system if initiated early in life.

Although epithelial cells are a source of soluble mediators, a burgeoning area of research concerns the identification of other sources of cytokines that promote $\mathrm{T}_{\mathrm{H}} 2$ responses. Several newly identified innate subsets in the gut and lung produce cytokines and can contribute to allergic responses. The challenge now is to dissect their relative importance in relation to the complex array of adaptive immune cells in the lung (such as $\mathrm{T}_{\mathrm{H}} 17, \mathrm{~T}_{\mathrm{H}} 9$ and regulatory $\mathrm{T}$ cells) that affect disease.

Nowhere is this complexity more apparent than in the clinic, as asthma does not present as a single disease entity. Rather, distinct phenotypes of asthma are identifiable, and these distinct cohorts respond differently to medications, a fact that may account for the recent failure of several targeted biologics that were tested in large, unselected patient populations. In a recent study, patients expressing higher serum concentrations of periostin, a protein released with activation of the IL-13 pathway, responded favorably to an interleukin-13-targeting antibody (N. Engl. J. Med. 365, 1088-1098, 2011). The hope is that, in the future, biomarkers reflecting activation of a pathogenic cascade may be used to stratify individuals before treatment to increase the likelihood of success. By combining unbiased genetic approaches and immunophenotyping of individuals, asthma may eventually be subcategorized into distinct endotypes on the basis of molecular mechanisms, opening the door to personalized therapy. The existence of multiple or mixed endotypes suggests that combination therapy may be needed to adequately control disease in subsets of individuals with asthma.

These and other advances have challenged previously held hypotheses regarding the origins of disease and are fostering new avenues of research. The newly uncovered targets suggest novel ways of intervening early, curbing the progression to chronic disease and managing disease in an underserved population. Despite setbacks in recent clinical trials, we are confident these findings will add to the armamentarium in the near future.

In closing, we thank Almirall and Theravance for their financial support to produce this special issue of the journal. As always, Nature Medicine takes full editorial responsibility for the content of these pages. 